\title{
Equilibria and Stability Around a Straight Rotating Segment with a Parabolic Profile of Mass Density
}

\author{
Nour-Eddine Najid* and El Haj Elourabi
}

Equipe Mécanique Statistique et Astronomie Laboratoire de Physique Théorique et Appliquée Université Hassan II Ain Chock- Faculté des Sciences Ain Chock BP 5366 Maarif Casablanca, Morocco

\begin{abstract}
In a previous work, we established the closet form of the potential generated by a massive inhomogeneous straight segment. We studied the dynamical behavior in the field of this segment at rest. Now, we plane to explore the case where the segment is in rotation around the axis perpendicular to the plane of study. We prove the existence of collinear and isosceles points of equilibrium. Their stability depend both on the rate of rotation as on the parameter governing the mass distribution of the parabolic profilE of density.
\end{abstract}

Keywords: Potential, Inhomogeneous distribution, Equilibrium, Asteroids, Stability.

\section{INTRODUCTION}

Many ways and methods were used to model the potential of irregular bodies. The main target for this is to study gravitational behavior, equilibrium and stability of a test particle around them. This subject is in fact an old/new one $[1,2]$. The discoveries of binary asteroids have opened this important field of research. Space missions to minor bodies in the solar system have piqued new interest in this subject. Many attempts have been made to estimate the potential. A harmonic polyhedron was used by Werner and Scheeres for asteroid 4769 Castalia [3, 4]. Ellipsoids, material points and a segment of double material were used by Bartczak and Breiter in [5] and Bartczak et al., [6], as the model of irregular elongated bodies. Riaguas et al., [7] proposed a homogeneous straight segment. Ellipe and Lara [8] described the motion around asteroid 433 Eros with the same homogeneous model. In a former work in our laboratory Najid et al., [9] studied and developed a new model in which we established the analytically closed expression of the potential generated by a massive inhomogeneous straight segment at rest. To be consistent with the geometrical shape, we used a parabolic profile of mass distribution. In our present work, we explore the case in which the inhomogeneous segment is rotating around an axis perpendicular to the plane of motion. In the first part, we establish the set of differential equations of motion in the synodical referential and we define the effective potential. The second part is devoted to the points of equilibrium, we prove the existence of two collinear points and isosceles others. In the third part, we study the linear stability. All over this work, we compare our results to the particular cases of homogeneous study made by Ellipe et al., [10] we prove the existence of two kinds of equilibrium depending on the rate of rotation, concerning the isosceles points, and the parameter governing the mass distribution of

*Address correspondence to this author at the Equipe Mécanique Statistique et Astronomie Laboratoire de Physique Théorique et Appliquée Université Hassan II Ain Chock- Faculté des Sciences Ain Chock BP 5366 Maarif Casablanca Morocco; Tel: 0665020353 ; Fax: 0522230674;

E-mail: ne.najid@gmail.com the parabolic profile of density. It is of great importance when exploring the formation of new structures around asteroids and even protoplanets.

\section{II-Dynamical Study}

We consider an inhomogeneous straight segment of length $2 I$ and mass $M$ which lies along the $\mathrm{x}$-axis, with a parabolic profile of density, expressed by $\lambda(x)=-a x^{2}+b$ in which $a$ and $b$ are linked by $a<\frac{b}{l^{2}}$ and $M=-\frac{2}{3} a l^{3}+2 b l$. The gravitational potential created by this one dimensional body at a certain point $P$ in the space may be expressed in closed form [9]

$U(P)=-\frac{G}{32 l^{2}}\left\{12 a l s d^{2}-16 a l^{3} s+\left[8 l^{2} a\left(s^{2}-2 p\right)-3 a s^{2} d^{2}-16 l^{4} a+32 b l^{2}\right]\right.$

$\left.\ln \left(\frac{s+2 l}{s-2 l}\right)\right\}$

as $s=r_{1}+r_{2}, d=r_{1}-r_{2}$ and $p=r_{1} \cdot r_{2}$, are auxiliary functions depending only on distances $r_{1}$ and $r_{2}$ of the particle to the end points of the segment. $\mathrm{G}$ is the gravitational constant. We suppose that the segment uniformly rotates with angular velocity $\omega$ about the Z-axis perpendicular to the segment and fixed in the space. We study the motion of a test particle $P$, with unit mass, in a synodic reference frame $(O x y z)$, with origin at the center of mass $O$ as shown in Fig. (1).

The Newton law in synodical referential $R$ yields

$\left(\frac{d^{2} \overrightarrow{O P}}{d t^{2}}\right)_{R}+2 \vec{\omega} \wedge\left(\frac{d \overrightarrow{O P}}{d t}\right)_{R}+\vec{\omega} \wedge(\vec{\omega} \wedge \overrightarrow{O P})=-\vec{\nabla} U(P)$

The projection of (2) in the synodical referential gives 


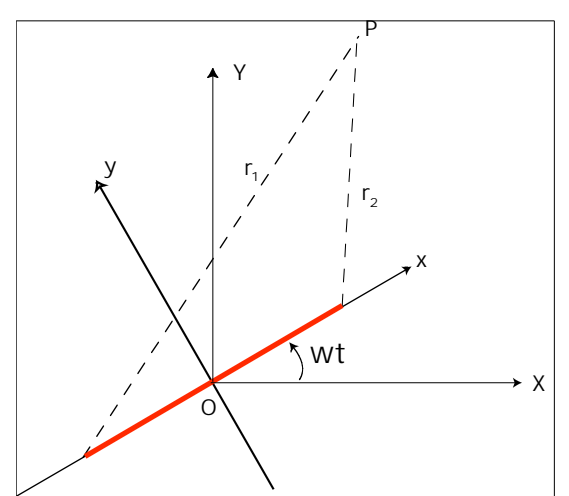

Fig. (1). Synodical referential.

$$
\left\{\begin{array}{l}
\ddot{x}-2 \omega \dot{y}=\omega^{2} x-\frac{\partial U}{\partial x} \\
\ddot{y}+2 \omega \dot{x}=\omega^{2} y-\frac{\partial U}{\partial y} \\
\ddot{z}=-\frac{\partial U}{\partial z}
\end{array}\right.
$$

By defining an effective potential $W$, we get

$$
W(x, y, z)=U(x, y, z)-\frac{\omega^{2}}{2}\left(x^{2}+y^{2}\right)
$$

In which $U(x, y, z)$ is expressed by (1). The system (3) then becomes

$$
\left\{\begin{array}{l}
\ddot{x}-2 \omega \dot{y}=\omega^{2} x-\frac{\partial U}{\partial x}=-W_{x} \\
\ddot{y}+2 \omega \dot{x}=\omega^{2} y-\frac{\partial U}{\partial y}=-W_{y} \\
\ddot{z}=-\frac{\partial U}{\partial z}=-W_{z}
\end{array}\right.
$$

With $W_{x}=\frac{\partial W}{\partial x}, W_{y}=\frac{\partial W}{\partial y}$ and $W_{z}=\frac{\partial W}{\partial z}$. The expressions (1) and (4) give

$$
\begin{aligned}
& W=-\frac{G}{32 l^{2}}\left\{12 a l s d^{2}-16 a l^{3} s+\left[8 l^{2} a\left(s^{2}-2 p\right)-3 a s^{2} d^{2}-\right.\right. \\
& \left.\left.16 l^{4} a+32 b l^{2}\right] \ln \left(\frac{s+2 l}{s-2 l}\right)\right\}-\frac{\omega^{2}}{2}\left(x^{2}+y^{2}\right)
\end{aligned}
$$




\section{II-1-Expressions of $W_{x}, W_{y}$ and $W_{z}$}

The expressions of $W_{x}, W_{y}$ and $W_{z}$ allow us to complete the system of differential equation governing the motion of the test particle. After a long and laborious calculation we reach

\section{II-2-Equations of Motion}

By substituting (7), (8) and (9) in the system (5), we obtain the equations of motion of the test particle

$$
\ddot{z}=\frac{G}{32 l^{2} p}\left\{\begin{array}{l}
32 a l^{2} z p \ln \left(\frac{s+2 l}{s-2 l}\right)-4 l a z s\left(3 d^{2}+4 l^{2}\right) \\
-\frac{4 l z s}{s^{2}-4 l^{2}}\left[8 l^{2} a\left(s^{2}-2 p\right)-3 a s^{2} d^{2}-16 a l^{4}+32 b l^{2}\right]
\end{array}\right\}
$$

The case of homogeneous profile of density, $a=0$, lead to the equations

$$
\left\{\begin{array}{l}
\ddot{x}-2 \omega \dot{y}=\omega^{2} x\left(1-\frac{2 G M}{s p \omega^{2}}\right) \\
\ddot{y}+2 \omega \dot{x}=\omega^{2} y\left(1-\frac{2 G M}{\omega^{2}} \frac{s}{p\left(s^{2}-4 l^{2}\right)}\right) \\
\ddot{z}=\frac{G}{32 l^{2} p}\left(-\frac{4 l z s}{s^{2}-4 l^{2}}\right) 32 b l^{2}
\end{array}\right.
$$

The use of the following scaling $t^{\prime}=t \omega$ and $r^{\prime}=\frac{r}{2 l}$, and the dimensionless parameter $k=\frac{G M}{(2 l)^{3} \omega^{2}}$ defined by the ratio of the gravitational acceleration to the centrifugal acceleration give the particular case as in [10]. The inhomogeneous straight rotating segments (10), (11) and (12) are strongly non linear and coupled. It needs a deep numerical treatment. In fact, it is out of question to plan to work it out in an analytical way.

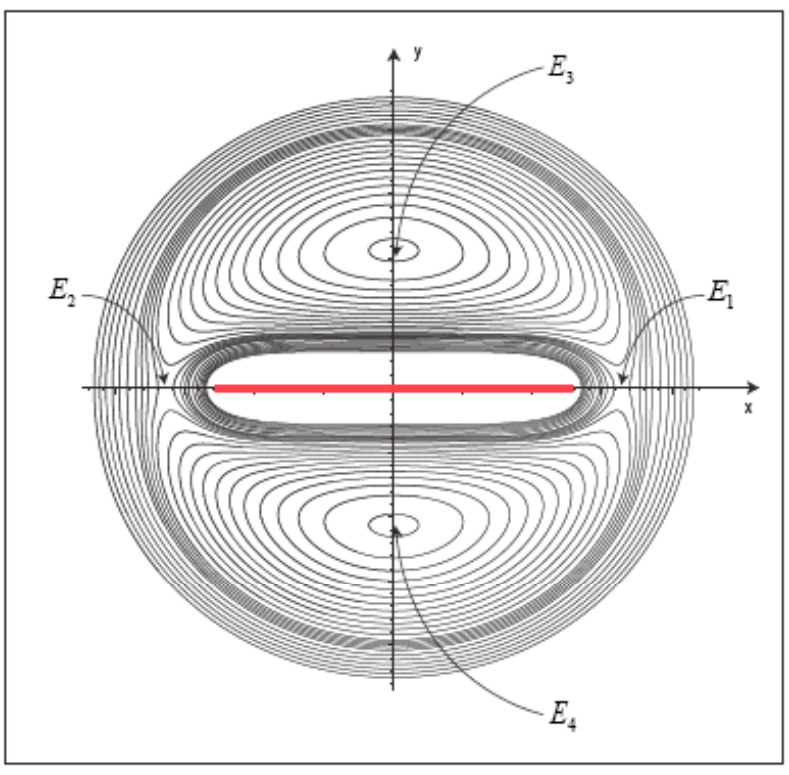

Fig. (2). Zero velocity curves on the (x,y) plane.

\section{III-THE EQUILIBRIA}

The dynamical system defined by equations (5) admit the Jacobi integral $C=2 W(x, y, z)+\left(\dot{x}^{2}+\dot{y}^{2}+\dot{z}^{2}\right)$. For a given set of initial conditions, the orbit must be inner $(2 W(x, y, z) \leq C)$ the surface of zero velocity expressed by $C=2 W(x, y, z)$. The equilibrium positions are given by solving the equations (10), (11) and (12). The conditions are: $\dot{x}=0, \ddot{x}=0, \quad \dot{y}=0, \quad \ddot{y}=0, \quad \dot{z}=0$ and $\ddot{z}=0$. The equation (12) implies that $z=0$, we will then restrict our study to the plane $(x, y)$. In this case then, we have $C=2 W(x, y, 0)$. The contour level of the surface given by $g(x, y, C)=2 W-C=0$ are shown in Fig. (2). We notice that we have four equilibrium positions: two of them, $E_{1}$ and $E_{2}$, are hyperbolic located along the x-axis and the two others, $E_{3}$ and $E_{4}$, are elliptical located along the y-axis. (10) and (11) are reduced to

$$
\frac{G a}{16 l^{2} p}\left\{\begin{array}{l}
2 l(x s-l d)\left(3 d^{2}-4 l^{2}\right)+12 l s d(l s-x d)+ \\
{\left[s(x s-l d)\left(8 l^{2}-3 d^{2}\right)-8 l^{2} x\left(s^{2}-2 p\right)+8 l^{3} s d-3 s^{2} d(l s-x d)\right] \times \ln \left(\frac{s+2 l}{s-2 l}\right)} \\
-\frac{2 l(x s-l d)}{s^{2}-4 l^{2}}\left[8 l^{2}\left(s^{2}-2 p\right)-3 s^{2} d^{2}-16 l^{4}+\frac{32 b l^{2}}{a}\right]
\end{array}\right\}+\omega^{2} x=0
$$

$$
\frac{G}{32 l^{2} p}\left\{\begin{array}{l}
32 l^{2} \text { ayp } \ln \left(\frac{s+2 l}{s-2 l}\right)-4 l a s y\left(3 d^{2}+4 l^{2}\right)- \\
\frac{4 y l s}{s^{2}-4 l^{2}}\left[8 l^{2} a\left(s^{2}-2 p\right)-3 a s^{2} d^{2}-16 a l^{4}+32 b l^{2}\right]
\end{array}\right\}+\omega^{2} y=0
$$




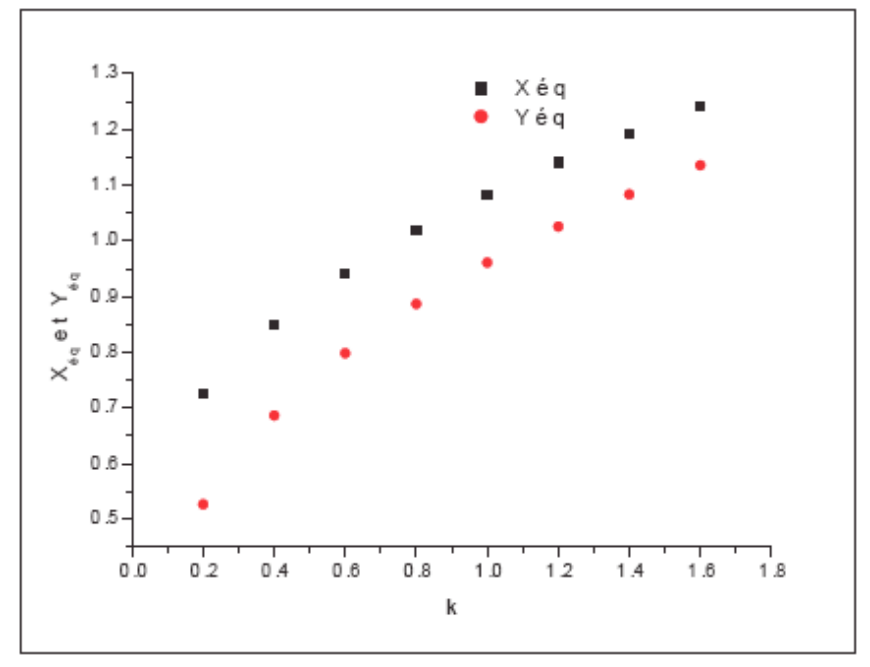

Fig. (3). Effect the ratio $\mathrm{k}$ on the equilibrium positions with $\mathrm{a}=0.5$, $\mathrm{b}=1$ and $\mathrm{k}=1$.

The equilibrium positions depend on the parameters $a$ and $\omega$. For a given rate of rotation $(\omega=$ cte $)$ and by varying the value of $a$, which act as a parameter of the profile of the density of the inhomogeneous straight segment, we establish the Table (1). We deduce after a close insight that when the gravitation is stronger the equilibrium positions along the $y-$ axis are farthest; this is to balance the centrifugal effect. In contrary, the equilibrium positions along $\mathrm{x}$-axis are less con- cerned. We can check the concordance with the values of homogeneous segment as in [10].

For a given value of a, and by varying the values of $\omega$, we have the Table 2 . We notice that when $\omega$ increases the two kinds of equilibrium positions become closer to the segment. This can be interpreted as an effect to balance the gravitation. Fig. (3). Shows the effect of both gravitation and rotation on the equilibrium positions.

\section{IV-LINEAR STABILITY}

In order to determine stability of the equilibrium points found above, one needs the variational equations of the system (5). We define a vector $\xi=(\delta x, \delta y, \delta \dot{x}, \delta \dot{y})$ as the variations of the vector space phase and the vector derivation $\dot{\xi}=(\delta \dot{x}, \delta \dot{y}, \delta \ddot{x}, \delta \ddot{y})$ [11]. The variational equations of the system (5) give:

$$
\dot{\xi}=[A] \xi=\left(\begin{array}{cccc}
0 & 0 & 1 & 0 \\
0 & 0 & 0 & 1 \\
-W_{x x} & -W_{x y} & 0 & 2 \\
-W_{y x} & -W_{y y} & -2 & 0
\end{array}\right)\left(\begin{array}{c}
\delta x \\
\delta y \\
\delta \dot{x} \\
\delta \dot{y}
\end{array}\right)
$$

Where: $W_{x x}=\frac{\partial W_{x}}{\partial x}, W_{x y}=\frac{\partial W_{x}}{\partial y}, W_{y x}=\frac{\partial W_{y}}{\partial x}$ and $W_{y y}=\frac{\partial W_{y}}{\partial y}$.

Table 1. Position's Equilibria for $\omega=1$ and Different Values of a

\begin{tabular}{|c|c|}
\hline \hline Value of a & $(\mathbf{x}, \mathbf{y})$ Coordinates of Equilibrium Positions \\
\hline \hline 0 & $(0,0.72),(0,-0.72),(0.95,0),(-0.95,0)$ \\
\hline 0.5 & $(0,0.714),(0,-0.714),(0.94,0),(-0.94,0)$ \\
\hline 1 & $(0,0.715),(0,-0.715),(0.936,0),(-0.936,0)$ \\
\hline 1.5 & $(0,0.715),(0,-0.715),(0.935,0),(-0.935,0)$ \\
\hline 2 & $(0,0.716),(0,-0.716),(0.934,0),(-0.934,0)$ \\
\hline 2.5 & $(0,0.716),(0,-0.716),(0.933,0),(-0.933,0)$ \\
\hline 3 & $(0,0.718),(0,-0.718),(0.932,0),(-0.932,0)$ \\
\hline 4 & $(0,0.719),(0,-0.719),(0.931,0),(-0.931,0)$ \\
\hline 5 & $(0,0.721),(0,-0.721),(0.930,0),(-0.930,0)$ \\
\hline
\end{tabular}

Table 2. Position's Equilibria for $\mathrm{a}=1$ and Different Values of $\omega$

\begin{tabular}{|c|l|}
\hline Value of $\omega$ & $(x, y)$ Coordinates of Equilibrium Positions \\
\hline \hline 0.1 & $(0,4.632),(0,-4.632),(2.153,0),(-2.153,0)$. \\
\hline 0.3 & $(0,2.213),(0,-2.213),(1.500,0),(-1.500,0)$. \\
\hline 1 & $(0,0.962),(0,-0.962),(1.038,0),(-1.038,0)$. \\
\hline 1.5 & $(0,0.715),(0,-0.715),(0.936,0),(-0.936,0)$. \\
\hline 2 & $(0,0.580),(0,-0.580),(0.880,0),(-0.880,0)$. \\
\hline 2.5 & $(0,0.482),(0,-0.482),(0.672,0),(-0.672,0)$. \\
\hline 3 & $(0,0.415),(0,-0.415),(0.568,0),(-0.568,0)$. \\
\hline 5 & $(0,0.267),(0,-0.267),(0.299,0),(-0.299,0)$. \\
\hline
\end{tabular}


Table 3. $a=0.5, b=5$ and $\omega$ is Variable

\begin{tabular}{|c|c|c|}
\hline Value of $\omega$ & $(x, y)$ Coordinates of $E_{1}$ & Eigenvalues \\
\hline \hline \multirow{2}{*}{0.5} & $(1.63,0)$ & $-0.5 .10^{-16}+1.5 . I ;-0.5 .10^{-16}-1.5 . I$ \\
& & $-0.2 .10^{-15}+0.4 . I ;-0.2 .10^{-15}-0.4 . I$ \\
\hline \multirow{2}{*}{1} & $(1.07,0)$ & $-0.26 .10^{-15}+1.1 . I ;-0.26 .10^{-15}-1.1 . I$ \\
& & $-0.27 .10^{-15}+0.66 . I ;-0.27 .10^{-15}-0.66 . I$ \\
\hline 1.5 & $(0.86,0)$ & $1.4,-1.4,1.9 . I,-1.9 . I$ \\
\hline 2 & $(0.75,0)$ & $2.21,-2.21,2.81 . I,-2.81 . I$ \\
\hline
\end{tabular}

Table 4. $a=1, b=5$ and $\omega$ is Variable

\begin{tabular}{|c|c|c|}
\hline Value of $\omega$ & $(x, y)$ Coordinates of $E_{1}$ & Eigenvalues \\
\hline \hline \multirow{2}{*}{0.5} & $(1.63,0)$ & $-0.2 .10^{-14}+0.9 . I ;-0.2 .10^{-14}-0.9 . I$ \\
& \multirow{2}{*}{$(1.45,0)$} & $0.16 .10^{-14}+0.4 . I ; 0.16 .10^{-14}-0.4 . I$ \\
\hline \multirow{2}{*}{0.6} & & $0.27 .10^{-15}+1.4 . I ; 0.27 .10^{-15}-1.4 . I$ \\
& \multirow{2}{*}{$(1.22,0)$} & $-0.9 .10^{-16}+0.4 . I ;-0.9 .10^{-16}-0.4 . I$ \\
\hline \multirow{2}{*}{0.8} & & $0.94 .10^{-15}+0.7 . I ; 0.94 .10^{-15}-0.7 . I$ \\
& \multirow{2}{*}{$1(1.07,0)$} & $-0.37 .10^{-15}+0.2 . I ;-0.37 .10^{-15}-0.2 . I$ \\
\hline
\end{tabular}

Table 5. $a=2, b=5$ and $\omega$ is Variable

\begin{tabular}{|c|c|c|}
\hline Value of $\omega$ & $(x, y)$ Coordinates of $E_{1}$ & Eigenvalues \\
\hline \hline \multirow{2}{*}{0.5} & $(1.63,0)$ & $0.9 .10^{-15}+0.9 . I ; 0.9 .10^{-15}-0.9 . I$ \\
& $(1.32,0)$ & $-0.52 .10^{-15}+0.4 . I ;-0.52 .10^{-15}-0.4 . I$ \\
\hline 0.7 & $(1.22,0)$ & $-0.1+0.6 . I ;-0.1-0.6 . I$ \\
& & $0.1+0.6 . \mathrm{I} ; 0.1-0.6 . \mathrm{I}$ \\
\hline 0.8 & $(1.07,0)$ & $0.1 .10^{-14}+0.7 . I ; 0.1 .10^{-14}-0.7 . I$ \\
& & $-0.8 .10^{-15}+0.2 . I ;-0.8 .10^{-15}-0.2 . I$ \\
\hline 1 & & $2.21,-2.21,2.81 . \mathrm{I},-2.81 . \mathrm{I}$ \\
\hline
\end{tabular}

Table 6. $a=0, b=0.5$ and $\omega$ is Variable

\begin{tabular}{|c|c|c|}
\hline Value of $\omega$ & $(x, y)$ Coordinates of $E_{1}$ & Eigenvalues \\
\hline \hline 0.5 & $(1.63,0)$ & $-0.15 .10^{-15}+1.9 . I ;-0.15 .10^{-15}-1.9 . I$ \\
& $(1.08,0)$ & $-0.33 ; 0.33$ \\
\hline 1 & $(0.84,0)$ & $1.33,-1.33,2.1 . I,-2.2 . I$ \\
\hline 1.6 & $(0.76,0)$ & $2.8,-2.8,2.8 . I,-2.8 . I$ \\
\hline 2 & $3.9,-3.9,3.5 . I,-3.5 . I$ \\
\hline
\end{tabular}

\section{IV-1-STABILITY OF THE COLLINEAR EQUILIBRIA $E_{1}$ AND $E_{2}$}

The problem is symmetric, the stability of the point $\mathrm{E}_{l}$ is the same as its symmetric $E_{2}$. The numerical results which appear in Tables (3), (4), and (5), show that for every inhomogeneous body $a \neq 0$ there is a critical value $\omega_{c}$, for which if $\omega<\omega_{c}$ all eigenvalues have null real part, hence the collinear equilibria are stable, and they are unstable for $\omega>\omega_{c}$. The Table (6) corresponds to the homogeneous body $\mathrm{a}=0$, we notice that the collinear equilibria are always unstable as in [10]. 
Table 7. $a=0.5, b=5$ and $\omega$ is Variable

\begin{tabular}{|c|c|c|}
\hline Value of $\omega$ & $(x, y)$ Coordinates of $E_{3}$ & Eigenvalues \\
\hline 0.5 & $(0,1.56)$ & $\begin{array}{c}-0.3 .10^{-15}+1.8 . I ;-0.3 .10^{-15}-1.8 . I \\
-0.6 .10^{-17}+0.15 . I ;-0.86 .10^{-17}-0.15 . I\end{array}$ \\
\hline 1 & $(0,0.96)$ & $\begin{array}{l}-0.8 .10^{-15}+1.2 . I ;-0.8 .10^{-15}-1.2 . I \\
0.83 .10^{-15}+0.7 . I ; 0.83 .10^{-15}-0.7 . I\end{array}$ \\
\hline 1.5 & $(0,0.71)$ & $\begin{array}{c}-0.9+0.9 . \mathrm{I} ;-0.9-0.9 . \mathrm{I} \\
0.9+0.9 . \mathrm{I} ; 0.9-0.9 . \mathrm{I}\end{array}$ \\
\hline 2 & $(0,0.57)$ & $\begin{array}{c}-1.4+0.9 . \mathrm{I} ;-1.4-0.9 . \mathrm{I} \\
1.4+0.9 . \mathrm{I} ; 1.4-0.9 . \mathrm{I}\end{array}$ \\
\hline
\end{tabular}

Table 8. $a=1, b=5$ and $\omega$ is Variable

\begin{tabular}{|c|c|c|}
\hline Value of $\omega$ & $(x, y)$ Coordinates of $E_{3}$ & Eigenvalues \\
\hline 0.5 & $(0,1.56)$ & $\begin{array}{c}0.1 .10^{-15}+1.6 . I ; 0.1 .10^{-15}-1.6 . I \\
-0.1 .10^{-15}+0.33 . I ;-0.1 .10^{-15}-0.33 . I\end{array}$ \\
\hline 0.6 & $(0,1.38)$ & $\begin{array}{c}0.2 .10^{-15}+1.4 . I ; 0.2 .10^{-15}-1.4 . I \\
-0.9 .10^{-16}+0.4 . I ;-0.9 .10^{-16}-0.4 . I\end{array}$ \\
\hline 0.8 & $(0,1.22)$ & $\begin{array}{c}0.9 .10^{-15}+0.7 . I ; 0.9 .10^{-15}-0.7 . I \\
-0.3 .10^{-15}+0.2 . I ;-0.3 .10^{-15}-0.2 . I\end{array}$ \\
\hline 1 & $(0,0.96)$ & $\begin{array}{c}-0.7+0.9 . \mathrm{I} ;-0.7-0.9 . \mathrm{I} \\
0.7+0.9 . \mathrm{I} ; 0.7-0.9 . \mathrm{I}\end{array}$ \\
\hline
\end{tabular}

Table 9. $a=2, b=5$ and $\omega$ is Variable

\begin{tabular}{|c|c|c|}
\hline Value of $\boldsymbol{\omega}$ & $(x, y)$ Coordinates of $\boldsymbol{E}_{3}$ & Eigenvalues \\
\hline \hline \multirow{2}{*}{0.5} & $(0,1.56)$ & $-0.4 .10^{-16}+1.6 . I ;-0.4 .10^{-16}-1.6 . I$ \\
& & $0.1 .10^{-15}+0.33 . I ; 0.1 .10^{-15}-0.33 . I$ \\
\hline \multirow{2}{*}{0.6} & $(0,1.38)$ & $-0.4 .10^{-15}+1.4 . I ;-0.4 .10^{-15}+1.4 . I$ \\
& & $0.2 .10^{-15}+0.4 . I ; 0.2 .10^{-15}-0.4 . I$ \\
\hline \multirow{2}{*}{0.8} & $(0,1.13)$ & $0.3 .10^{-14}+0.7 . I ; 0.3 .10^{-14}-0.7 . I$ \\
& & $-0.8 .10^{-15}+0.2 . I ;-0.8 .10^{-15}-0.2 . I$ \\
\hline \multirow{2}{*}{1} & $(0,0.96)$ & $-0.7+0.9 . I ;-0.7-0.9 . I$ \\
\end{tabular}

\section{IV-2-STABILITY OF THE ISOSCELES EQUILIBRIA} $E_{3}$ AND $E_{4}$

The problem is symmetric, the stability of the point $E_{3}$ is the same as its symmetric $E_{4}$. The numerical results which appear in Tables (7), (8) and (9), show that for every inho- mogeneous body $a \neq 0$ there is a critical value $\omega_{c}$, for which if $\omega<\omega_{c}$ all eigenvalues have null real part, hence the isosceles equilibria are stable, and they are unstable for $\omega>\omega_{c}$. The table (10) shows the results of the particular case as in [10]. 
Table 10. $a=0, b=0.5$ and $\omega$ is Variable

\begin{tabular}{|c|c|c|}
\hline Value of $\omega$ & $(\boldsymbol{x}, \boldsymbol{y})$ Coordinates of $\boldsymbol{E}_{3}$ & Eigenvalues \\
\hline \hline \multirow{2}{*}{0.5} & $(0,1.56)$ & $0.2 .10^{-15}+1.9 . I ; 0.2 .10^{-15}-1.9 . I$ \\
& $0.4 .10^{-17}+0.03 . I ; 0.4 .10^{-17}-0.03 . I$ \\
\hline \multirow{2}{*}{1} & $(0,0.96)$ & $0.4 .10^{-15}+1.7 . I ; 0.4 .10^{-15}-1.7 . I$ \\
& & $-0.13 .10^{-15}+0.2 . I ;-0.13 .10^{-15}-0.2 . I$ \\
\hline \multirow{2}{*}{1.5} & $(0,0.71)$ & $-0.3+0.9 . \mathrm{I} ;-0.3-0.9 . \mathrm{I}$ \\
& & $0.3+0.9 . \mathrm{I} ; 0.3-0.9 . \mathrm{I}$ \\
\hline \multirow{2}{*}{2} & $(0,0.57)$ & $-0.9+0.9 . \mathrm{I} ;-0.9-0.9 . \mathrm{I}$ \\
& & $0.9+0.9 . \mathrm{I} ; 0.9-0.9 . \mathrm{I}$ \\
\hline
\end{tabular}

\section{CONCLUSION}

On this work we studied the gravitational behavior of a particle of unimportant mass in the field of an rotating inhomogeneous straight segment. The segment is modelised by a parabolic profile of mass density. For the analytical expression of the potential we found four positions of equilibrium, two of them are collinear located along the axis of the segment, they are hyperbolic. The two others are isosceles located symmetrically with respect to the segment along its mediatrix, they are elliptical. The new result concerning this analytical potential for the inhomogeneous segment, corresponds to the stable region for the collinear points. That is the difference in the cas of homogeneous segment as in [10]. This situation could explain the existence of halo around the segment like Gegenschein effect [12].

The main interest to study the location of equilibrium points is similar to that of Lagrangian points in three body problem. To explore irregular bodies, it is an opportunity to have equilibrium positions around them. In this way many spatial missions, like NEAR and ROSETTA were fulfilled by space agencies such as ESA and NASA [13] and [14]. As an extension of this work, we are planing to explore the case of a more detailed mass distribution. In fact many asteroids are far of a regular or symmetrical shape.

\section{CONFLICT OF INTEREST}

None declared.

\section{ACKNOWLEDGEMENT}

None declared.

\section{REFERENCES}

[1] MacRobert TM. Spherical harmonics London: Methnen and Co. LTD 1927.

[2] Kellogg OD. Foundations of Potential Theory. UK: Frederick Ungar Publishing Company 1954.

[3] Werner Robert A. The gravitational potential of homogeneous polyhedron or don't cut corners. Celestial Mech Dyn Astron 1994; 59: 253-78.

[4] Werne RA, Scheeres DJ. Exterior gravitation of polyhedron with harmonic and mascon gravitation representations of asteroid 4769 castalia. Celestial Mech Dyn Astron 1997; 65: 313-44.

[5] Breiter S, Bartczak P. Double material segment as the model of irregular bodies. Celestial Mech Dyn Astron 2003; 86: 131-41.

[6] Bartczak P, Breiter S, Jusiel P. Ellipsoids, material points and material segments. Celestial Mech Dyn Astron 2006; 96: 31-48.

[7] Riaguas A, Ellipe A, Lara M. Periodic orbits around a massive straight segment. Celestial Mech Dyn Astron 1999; 73: 169-78.

[8] Ellipe A, Lara M. A Simple Model for the Chaotic Motion Around (433) Eros. J Astronaut Sci 2003; 51: 391.

[9] Najid NE, Elourabi E, Zegoumou M. Potential generated by a massive inhomogeneous straight segment. Res Astron Astrophys 2011; 11(3): 345-52.

[10] Ellipe A, Lara M, Riaguas A. Periodic orbits around natural elongated bodies. RBCM-J. Braz Soc Mech Sci 1999; XXI: 198-207.

[11] Murray CD, Dermott SF. Solar system dynamics. UK: Cambridge 1999.

[12] Moulton FR. An introduction to celestial mechanics. New York: The Macmillan Company 1923.

[13] NEAR. Special Issue on the NEAR Mission to 433 Eros. J Astronaut Sci 1995; 43: 477.

[14] Schwehm G, Hechler M. Rosetta -ESA's planetary cornestone mission. ESA Bull 1994; 77: 7-18.

(c) Najid and Elourabi; Licensee Bentham Open.

This is an open access article licensed under the terms of the Creative Commons Attribution Non-Commercial License (http://creativecommons.org/licenses/by-nc/3.0/) which permits unrestricted, non-commercial use, distribution and reproduction in any medium, provided the work is properly cited. 